\title{
A Novel Approach to Extract Morphological Variables in Crystalline Polymers from Time-Resolved Synchrotron SAXS Data
}

\author{
Benjamin S. Hsiao*† and Ravi K. Vermał \\ DuPont Central Research and Development, Experimental Station, Wilmington, DE 19880- \\ 0302, USA.E-mail: bhsiao@sunysb.edu
}

(Received 15 February 1997; accepted 9 July 1997)

\begin{abstract}
A novel approach to extract morphological variables in crystalline polymers from time-resolved SAXS data using the method of correlation and interface distribution functions has been devised. The principle of the calculation is based on two alternative expressions of Porod's law using the form of the interference function. The approach enables the continuous estimate of the Porod constant, and corrections for liquid scattering and finite interface between the two phases, from the time-resolved data. A model of lamellar morphology has been implemented to interpret the calculated correlation and interface distribution functions. Many detailed morphological variables such as lamellar long period, thicknesses of crystal and amorphous phases, interface thickness, and scattering invariant can be estimated. An example analysis of isothermal crystallization in PET measured by synchrotron SAXS is demonstrated.
\end{abstract}

Keywords: time-resolved SAXS; correlation function; interface distribution function; PET; interference function; Porod's law; lamellar morphology; crystalline polymers.

\section{Introduction}

Small-angle scattering (via X-ray or neutron) techniques are frequently used to characterize the morphological information $(50-2000 \AA)$ in crystalline polymers. Very often, the information can be extracted only after some complicated data analysis involving the use of the correlation function (Debye \& Bueche, 1949; Debye, Anderson \& Brauberger, 1957; Vonk \& Kortleve, 1967) and the interface distribution function (Ruland, 1977). Calculation of these functions requires knowledge of Porod's law parameters at high scattering angles (Porod, 1951, 1952a,b) and the interpretation requires the assumption of a suitable morphological model (Glatter, 1982).

In the case of time-resolved small-angle scattering experiments, particularly with the use of synchrotron $X$-rays (SAXS), calculations of correlation and interface distribution functions from the measured intensities are usually difficult. The reasons are as follows. (i) A good signal-to-noise ratio is usually not available in the Porod region (at high scattering angles). This is because the quality of the data is often compromised by the aim of obtaining decent time resolution. Large noise leads to less confidence in determining the Porod parameters. In addition, the conventional method for estimating the Porod parameters employs a plot of $I q^{4}$ versus $q^{4}[q=(4 \pi / \lambda) \sin \theta]$, which can

\footnotetext{
+ Current address: Department of Chemistry, State University of New York at Stony Brook, Stony Brook, NY 11794-3400, USA.

$\ddagger$ Current address: Department of Chemical Engineering, California Institute of Technology, Pasadena, CA 91125 , USA.
}

significantly exaggerate the problem of large noise at high $q$ values. (ii) At some incidence the Porod region is simply not measured in the full range. The truncation problem is often incurred by the limited detector size, and can severely affect the calculation of these functions. As a result, most timeresolved synchrotron SAXS studies only report apparent profile changes (long period and invariant). Very few provide a detailed analysis using correlation and interface distribution functions.

In this work we present a novel approach for the calculation of the correlation and interface distribution functions from time-resolved synchrotron SAXS data. The calculation is made possible by using two alternative expressions of Porod's law to determine the Porod parameters and to correct the liquid scattering and finite interface between the scattering phases. Both expressions use forms of the interference function (Ruland, 1977) that are markedly different from the conventional method. We found that the proposed approach is capable of handling the data with low signal-to-noise ratios and/or limited Porod range. A lamellar model has also been implemented to analyze the calculated functions, which reveal morphological variables such as long period, lamellar thicknesses of both constituting phases, interface thickness and invariant. This analysis is suitable for crystalline polymers.

\section{Theoretical background}

The relationships for calculating the one-dimensional correlation function, $\gamma_{1}(r)$, interface distribution function, 
$g_{1}(r)$, and interference function, $G_{1}(q)$, using the scattering intensity measured from a pinhole, slit or fiber geometry are summarized as follows. This intensity must be free of contributions from liquid scattering and finite interface between the constituting phases. Detailed procedures for obtaining this intensity from the measured intensity, $I_{\text {obs }}$, will be discussed in the following section.

\subsection{Pinhole geometry}

The scattering intensity, $I(q)$, measured from the isotropic three-dimensional object using a pinhole geometry can be transformed to the one-dimensional intensity function, $I_{1}(q)$, by Lorentz correction (Baltá-Calleja \& Vonk, 1989),

$$
I_{1}(q)=c /(q) q^{2},
$$

where $c$ is a constant. In this case the correlation and interface distribution functions become (Vonk \& Kortleve, 1967; Vonk, 1973; Ruland, 1977; Stribeck \& Ruland, 1978)

$$
\begin{gathered}
\gamma_{1}(r)=\left[\int_{0}^{\infty} I_{1}(q) \cos (q r) \mathrm{d} q\right] / Q \\
g_{1}(r)=\partial^{2}\left[\gamma_{1}(r)\right] / \partial r^{2}=\left[-\int_{0}^{\alpha} I_{1}(q) q^{2} \cos (q r) \mathrm{d} q\right] / Q
\end{gathered}
$$

where $Q$ is the invariant defined as

$$
Q=\int_{0}^{\infty} I_{1}(q) \mathrm{d} q
$$

The interference function, $G_{1}(q)$, is the Fourier transform of the interface distribution function (Ruland, 1977)

$$
g_{1}(r)=\int_{0}^{\infty} G_{1}(q) \cos (q r) \mathrm{d} q
$$

In the above equation $G_{1}(q)$ can also be expressed as

$$
G_{1}(q)=\lim _{q \rightarrow \infty} I_{1}(q) q^{2}-I_{1}(q) q^{2} .
$$

\subsection{Slit geometry}

If a slit geometry is used, the measured intensity, $\bar{I}(q)$, may be considered as being smeared by a slit of infinite height (approximately). The above functions thus become (Ruland, 1977; Stribeck \& Ruland, 1978)

$$
\begin{aligned}
\gamma_{1}(r)= & \left\{\int_{0}^{\infty} \bar{I}(q) q\left[J_{0}(q r)-(q r) J_{1}(q r)\right] \mathrm{d} q\right\} / Q, \\
g_{1}(r)= & \left(\int _ { 0 } ^ { \infty } \overline { G } _ { 1 } ( q ) \left\{3\left[J_{0}(q r)-J_{2}(q r)\right]+(q r)\left[J_{3}(q r)\right.\right.\right. \\
& \left.\left.\left.-3 J_{1}(q r)\right] / 2\right\} \mathrm{~d} q\right) / Q,
\end{aligned}
$$

where $J_{n}$ is a Bessel function of order $n, Q$ is the invariant,

$$
Q=\int_{0}^{\infty} \bar{I}(q) q \mathrm{~d} q
$$

and the interference function, $G_{1}(q)$, can be calculated from

$$
\bar{G}_{1}(q)=\lim _{q \rightarrow \infty} \bar{I}(q) q^{3}-\bar{I}(q) q^{3} .
$$

\subsection{Fiber geometry}

In a highly oriented system, such as high module fiber or film, the sliced intensity, $I(q)$, along the meridional direction is similar to the one-dimensional integrated intensity profile, $I_{1}(q)$ (Hsiao et al., 1995). In this case, equations (2)-(6) can be applied directly with the sliced intensity without Lorentz correction.

\subsection{Data extrapolation}

In the above expressions all integrations have to be carried out over the range $0 \leq q \leq \infty$. Experimentally this is not feasible since both limits are beyond the resolution of any detector. If the limits for the detector are $q_{1}$ and $q_{2}$ (larger value), the integration can be divided into three parts. For example, the invariant $Q$ [equation (4)] can be written as (Russell, 1991)

$$
Q=\int_{0}^{q_{1}} I(q) q^{2} \mathrm{~d} q+\int_{q_{1}}^{q_{p}} I(q) q^{2} \mathrm{~d} q+\int_{q_{p}}^{\infty} I(q) q^{2} \mathrm{~d} q .
$$

The first integral is the area of a triangle with base $q_{1}$ and $I\left(q_{1}\right) q_{1}{ }^{2}$, the second integral is the numerical integral of the experimental data, and the third integral can be calculated by Porod's law with $q_{p}$ being the starting value of the Porod region $\left(q_{p}<q_{2}\right)$. In the Porod region the scattering intensity has the relationship (Porod's law; Porod, 1951, 1952a,b)

$$
\lim _{q \rightarrow \infty} I(q)=K / q^{4}
$$

where $K$ is the Porod constant. Thus (4) can be written as

$$
Q=\frac{1}{2} I\left(q_{1}\right) q_{1}^{3}+\int_{q_{1}}^{q_{p}} I(q) q^{2} \mathrm{~d} q+K / q_{p}
$$

The integrations in (2)-(9) are all carried out in a similar manner. The first two integrals are straightforward. The last integral requires an estimate of the Porod constant, which is obtained by an alternative method.

\section{Alternative approach for Porod analysis}

The typical measured scattering intensity, $I_{\mathrm{obs}}(q)$, includes the contributions from liquid-like 'background' scattering, $I_{b}(q)$, and the finite interface between the two constituting phases. According to Porod's law this intensity can be 
expressed by (Koberstein, Morra \& Stein, 1980)

$$
\lim _{q \rightarrow \infty} I_{\mathrm{obs}}(q)=I_{h}(q)+\left(K / q^{4}\right) \exp \left(-\sigma^{2} q^{2}\right)
$$

where $\sigma$ is related to the width of the interface. Instead of using the conventional method (an $I_{\text {obs }} q^{4}$ versus $q^{4}$ plot; see Glatter, 1982; Baltá-Calleja \& Vonk, 1989) we propose to use the following two alternative expressions of Porod's law (in the form of interference functions) to calculate the Porod parameters

$$
\begin{aligned}
\lim _{q \rightarrow \infty} G_{1}(q) & =\lim _{q \rightarrow \infty}\left\{K-\left[I_{\mathrm{obs}}(q)-I_{b}(q)\right] q^{4} \exp \left(\sigma^{2} q^{2}\right)\right\} \\
& =0 \\
\int_{0}^{\infty} G_{1}(q) \mathrm{d} q & =\int_{0}^{\infty}\left\{K-\left[I_{\mathrm{obs}}(q)-I_{b}(q)\right] q^{4} \exp \left(\sigma^{2} q^{2}\right)\right\} \mathrm{d} q \\
& =0 .
\end{aligned}
$$

Equation (16) is derived on the basis that if the presence of a finite transition zone between the two constituting phases has been accounted for, then the interface distribution function should start from the origin $\left[g_{1}(0)=0\right]$. This is because $g_{1}(r)$ is the Fourier transform of $G_{1}(q)$, the total area of the $G_{1}(q)$ versus $q$ plot is zero.

Thus, we can define the following two residual terms from (15) and (16),

$$
\begin{aligned}
& \sum \aleph_{1}^{2}=\sum_{q=q_{p}}^{q_{2}}\left(\left\{K-\left[I_{\mathrm{obs}}(q)-I_{b}(q)\right] q^{4} \exp \left(\sigma^{2} q^{2}\right)\right\}^{2}\right) \\
& \sum \aleph_{2}^{2}=\left(\sum_{q=0}^{q_{2}}\left\{K-\left[I_{\mathrm{obs}}(q)-I_{b}(q)\right] q^{4} \exp \left(\sigma^{2} q^{2}\right)\right\}\right)^{2} .
\end{aligned}
$$

If liquid scattering can be assumed as an even-powered polynomial function (Ruland, 1977; Wendorff \& Fisher, 1973a,b; Rathje \& Ruland, 1976)

$$
I_{b}(q)=a+b q^{2}+c q^{4}+d q^{6}
$$

we can minimize the following equation to obtain the values for parameters $(K, \sigma, a, b, c, d)$,

$$
\sum \aleph_{\text {sum }}^{2}=w_{1} \sum \aleph_{1}^{2}+w_{2} \sum \aleph_{2}^{2},
$$

where $w_{1}$ and $w_{2}$ are the fractions of two residues.

\section{Data smoothing}

It is found that noise migration in the Porod region by the alternative approach is considerably less than by the conventional method ( $I q^{4}$ versus $\left.q^{4}\right)$. However, for measurements involving a short acquisition time, the signal-to-noise ratio may still be too low to warrant a successful analysis. In this case noise reduction has to be implemented. We recommend the following smoothing procedure but caution readers to take two points into consideration. (i) The procedure should not introduce any artifacts in the Porod region. (ii) More smoothing is required at higher angles where the signal-to-noise ratio is lower. Our recommended smoothing procedure is as follows. For a linear array of points $\left(y_{1}, y_{2}, y_{3} \ldots y_{i} \ldots y_{n}\right)$, the smoothed array is taken as

$$
Y_{i}=\frac{Y_{i-n}+Y_{i-n+1}+\ldots+Y_{i-1}+y_{i+1}+y_{i+2}+\ldots+y_{i+n}}{2 n+1}
$$

where $Y$ refers to the value of $y$ in the smoothed array. For consideration (ii), the value of $n$ can be continuously increased with scattering angle. One way to implement this is to have $n$ vary as

$$
n=a_{0}+a_{1} q,
$$

where $a_{0}$ and $a_{1}$ are two arbitrary constants. If both constants are zero, there will be no smoothing applied.

\section{Calculation and analysis of correlation and interface distribution functions}

The selection of the Porod region is extremely sensitive to the accuracy of the calculation for correlation and interface distribution functions. Usually we begin the analysis with a transitional SAXS profile showing a distinctive scattering maximum. The range for the Porod region will be adjusted interactively such that the condition of (20) can be minimized in this profile. Once the limits for the Porod region are determined, the same values will be applied for all scattering profiles. In this regard we assume that the Porod region is identical for all scattering profiles. After the analysis involving (17)-(20) is carried out, the parameters $K, a, b, c, d$ and $\sigma$ can be obtained. The corresponding correlation and interface distribution functions are thus calculated using (2)-(6). There is a point of caution to be addressed here: any uncertainty in the Porod region of the interference function will manifest itself as Fourier ripples in the resultant interface distribution function. (Such an effect is usually negligible in the correlation function.) This problem can be minimized by choosing a narrower range of the Porod region to reduce the uncertainty in the interference function.

Lamellar morphology variables can be estimated from the correlation and interface distribution functions using a twophase model. This analysis is suitable for most crystalline polymers and may also be for some block polymers. The analysis of the correlation function by the two-phase model has been demonstrated in detail previously (Vonk, 1973; Strobl \& Schneider, 1980; Santa Cruz, Stribeck, Zachmann \& Baltá-Calleja, 1991). The thicknesses of the two constituent phases (crystal and amorphous) can be extracted by several approaches described by Strobl \& Schneider (1980). For example, one approach is based on the following relationship,

$$
x_{1} x_{2}=B / L_{c}^{M}
$$


where $x_{1}$ and $x_{2}$ are the linear fractions of the two phases within the lamellar morphology, $B$ is the value of the abscissa when the ordinate first equals zero in $\gamma_{1}(r)$, and $L_{c}^{M}$ represents the long period determined as the first maximum of $\gamma_{1}(r)$. Although this analysis reveals two thicknesses of the constituent phases, it cannot distinguish which represents the lamellar crystal or amorphous thickness. Information from other techniques [transmission electron microscopy (TEM), wide-angle X-ray diffraction (WAXD), differential scanning calorimetry (DSC) and density] is required for this purpose.

Analysis of the interface distribution function, $g_{1}(r)$, is relatively straightforward. The profile of $g_{1}(r)$ can be directly calculated from the Fourier transformation of the interference function or by taking the second derivative of the correlation function. In the physical sense the interface distribution function represents the probability of finding an interface along the density profile. A positive value indicates an even number of interfaces within a real space distance with respect to the origin. A negative value indicates an odd number of interfaces within the corresponding distance. In the case of lamellar morphology, odd numbers of interfaces correspond to integral numbers of long period. The shape of the probability distribution with distance for a given interface is manifested as the shape of the corresponding peak on the interface distribution function. These distributions can be deconvoluted to reveal more detailed morphological parameters (Stribeck, 1993).

In general, we find that the long-period values calculated from different methods, such as conventional analysis by Bragg's law, correlation function and interface distribution function, are quite different. However, their trends as functions of time and temperature are usually similar. The ordering of these long periods indicates the heterogeneity of the lamellar distributions in the morphology. This issue has been addressed previously (Santa Cruz et al., 1991; Verma \& Hsiao, 1996).

\section{Isothermal crystallization of poly(ethylene terephthalate)}

The chosen example for this study is the time-resolved synchrotron SAXS measurement during isothermal crystallization of poly(ethylene terephthalate) (PET). Measurements were carried out at the $\mathrm{X} 3 \mathrm{~A} 2$ beamline at the National Synchrotron Light Source, Brookhaven National Laboratory. The experimental conditions included the use of a pinhole collimator (Chu et al., 1994), a one-dimensional position-sensitive wire detector (MBruan), $1.54 \AA$ wavelength and a dual-temperature jump cell (Song et al., 1988). The PET sample was first equilibrated above its melting point $(553 \mathrm{~K})$ for $5 \mathrm{~min}$ and then rapidly cooled to four different temperatures $(493,503,513$ and $523 \mathrm{~K})$ for measurement. The equilibration time to reach the measured temperature was about $60 \mathrm{~s}$. Two sets of collection times were used: the first 48 frames were collected every $5 \mathrm{~s}$, the last 52 frames were collected every $30 \mathrm{~s}$.
Typical time-resolved SAXS profiles after the removal of background scattering (air and windows) at $503 \mathrm{~K}$ are illustrated in Fig. 1. Two distinguishable stages are seen, i.e. the intensity in the second stage is about six times higher than that of the first stage, which correspond well with the two collection times used. It is seen that crystallization begins at the first stage of the measurement. We have chosen the Porod region to be $0.09<q<0.14 \AA^{-1}$ for the calculation. The resultant Lorentz-corrected intensity profile ( $I q^{2}$ versus $q$, free of contributions by liquid scattering and finite interface boundary), interference function, $G(q)$, correlation function, $\gamma(r)$, and interface distribution function, $g(r)$, are illustrated in Figs. 2-5, respectively. These profiles reveal many subtle changes during isothermal crystallization of PET. In Fig. 3 the values of the interference function in the Porod region are near to zero, which bears the characteristics of the single maximum in the scattering profile of PET. In the case of block copolymers or other crystalline polymers (such as PE and PEO) where multiple SAXS maxima (due to higher orders of scattering) are detected, the behavior of the interference function in the

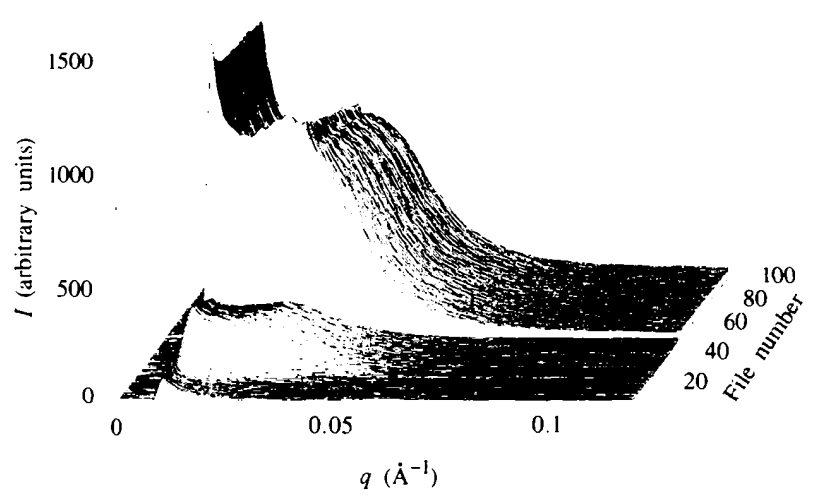

Figure 1

Time-resolved SAXS profiles during isothermal crystallization $(503 \mathrm{~K})$ of PET. The first 48 scans were collected with $5 \mathrm{~s}$ scan time, the last 52 scans were collected with $30 \mathrm{~s}$ scan time. The 'wiggles' in the scattering profiles are caused by smoothing using equation (22) with $a_{0}=1, a_{1}=5$.

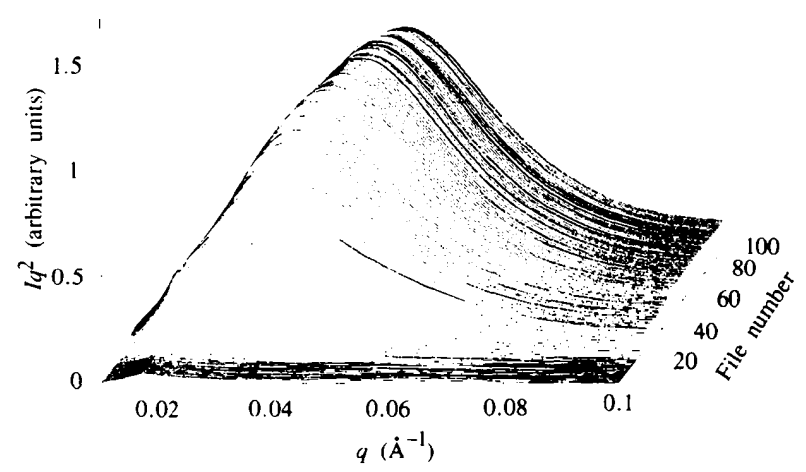

Figure 2

Lorentz-corrected intensity profiles calculated from data in Fig. 1. The Porod region was chosen as $0.09<q<0.14 \AA^{-1}$ for all analyses. 
Porod region exhibits an oscillation with decay along the abscissa.

Morphological results extracted by the two-phase lamellar model are shown in Figs. 6 and 7, respectively. It is seen that all three long periods calculated by Bragg's law, the correlation function and the interface distribution function are different $\left(L_{\mathrm{B}}>L_{c}^{M}>L_{l}\right)$. The trends in $L_{\mathrm{B}}$ and $L_{c}^{M}$ are similar, decreasing with time, but the value of $L_{I}$ remains almost unchanged. This relationship indicates that the

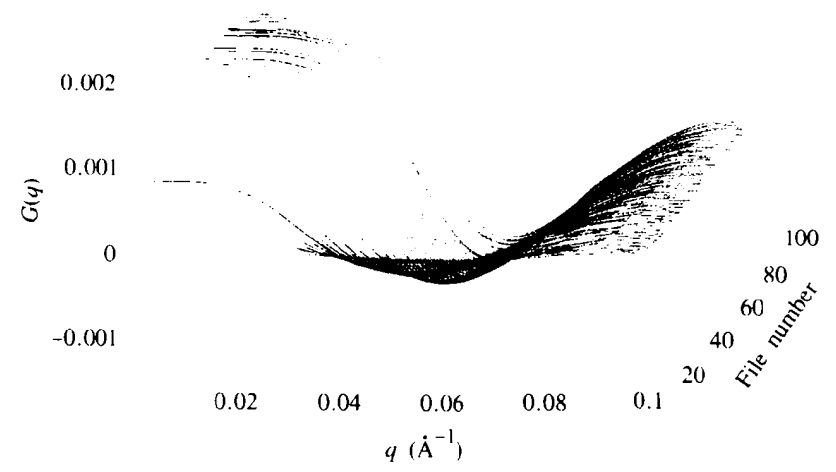

Figure 3

Calculated time-resolved interference functions, $G(q)$, from Fig. 1 .

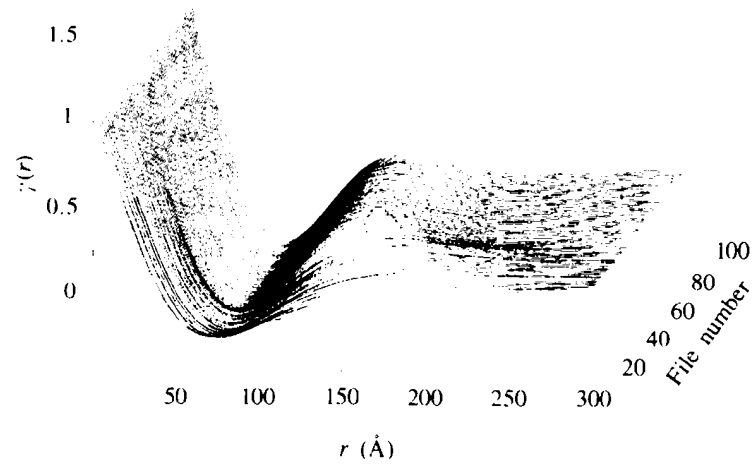

Figure 4

Calculated correlation functions, $\gamma(r)$ (normalized by the invariant $Q$ ), from Fig. 1.

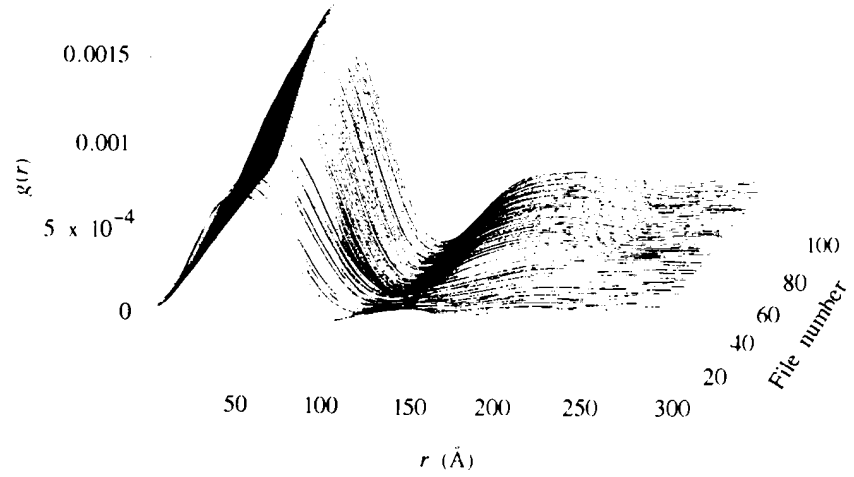

Figure 5

Calculated interface distribution functions, $g(r)$ (normalized by the invariant $Q$ ), from Fig. 1 . morphological development during PET isothermal crystallization deviates from the ideal two-phase model (Santa Cruz et al., 1991; Verma \& Hsiao, 1996). We have interpreted this behavior as due to the space-filling of thinner secondary crystal lamellae after the initial formation of thicker primary lamellae during isothermal crystallization of semi-stiff-chain crystalline polymers (Hsiao et al., 1995). The mass-average lamellar thicknesses of crystalline and amorphous phases can also be estimated from the correlation function (and from the interface distribution function, which is not discussed here due to its complexity for interpretation). The two calculated thicknesses along with the invariant are shown in Fig. 7. As indicated earlier, from the calculation itself it is impossible to determine which thickness represents the crystalline one. We have often relied on other methods (such as TEM and WAXD) and determine that the larger value in this study is the crystalline thickness. The detailed analysis and physical implications of these variables have been discussed by several authors (Santa Cruz et al., 1991; Stribeck, 1993; Verma \& Hsiao, 1996).

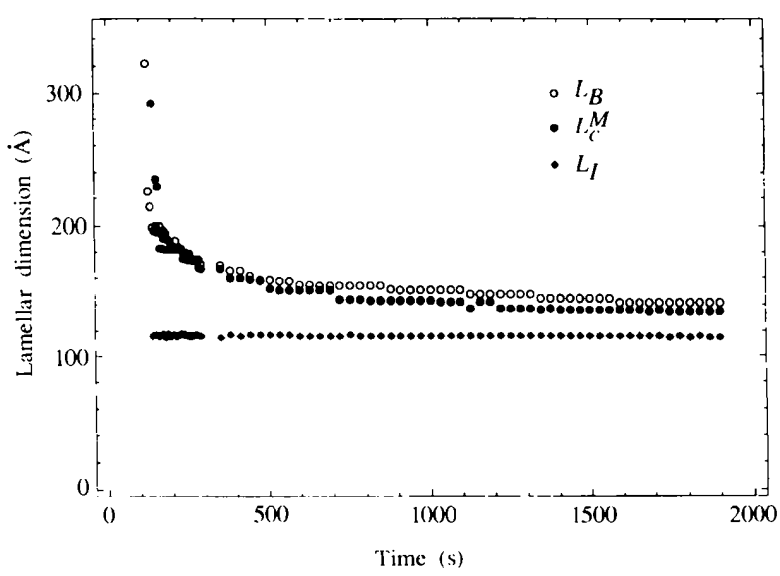

Figure 6

Comparison of long periods calculated using Bragg's law, $L_{\mathrm{B}}$, the correlation function, $L_{c}^{M}$, and the interface distribution function, $L_{l}$.

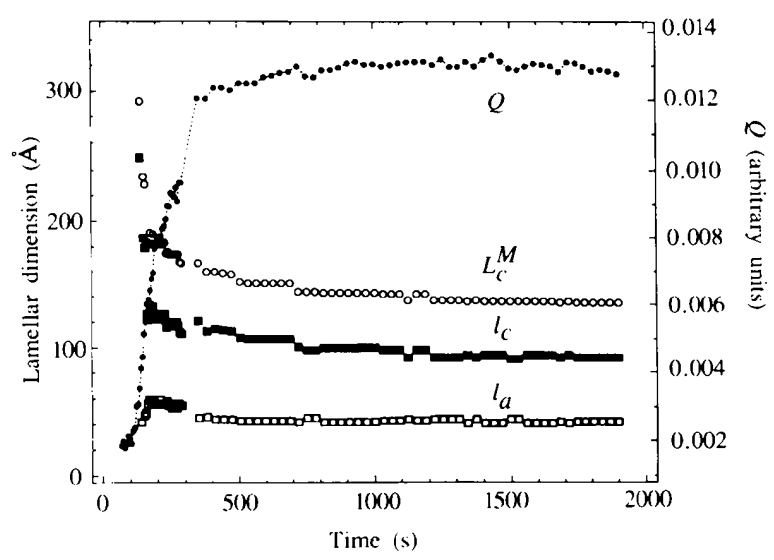

Figure 7

Lamellar morphological variables extracted from the correlation functions in Fig. 4: invariant $Q$, long period $L_{c}^{M}$, crystal lamellar thickness, $l_{c}$, and interlamellar amorphous thickness, $l_{a}$. 
In fact, the identification of the two calculated thicknesses can also be made by evaluating the effect of the crystallization temperature, $T_{c}$. The values of $L_{c}^{M}, l_{1}$ and $l_{2}$ as a function of $T_{c}$ are shown in Figs. 8(a)-8(c). In Fig. 8(a) the value of $L_{c}^{M}$ is seen to increase with increasing $T_{c}$. This

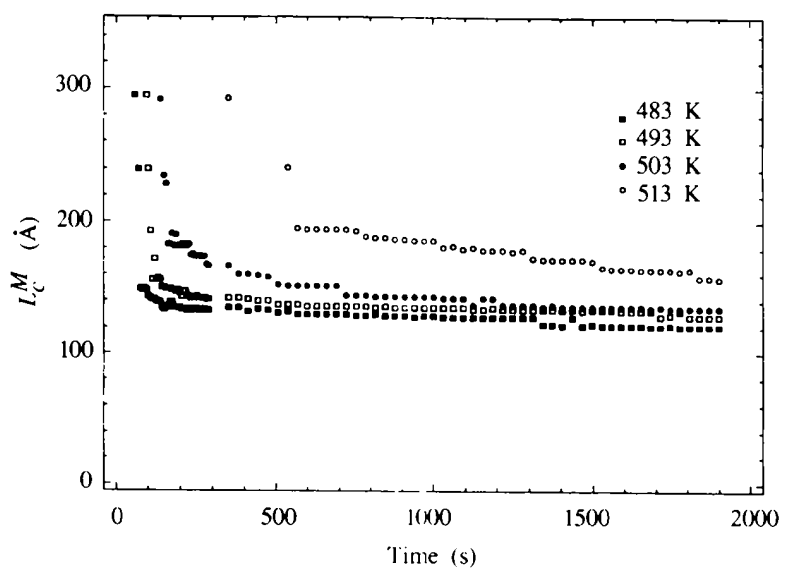

(a)

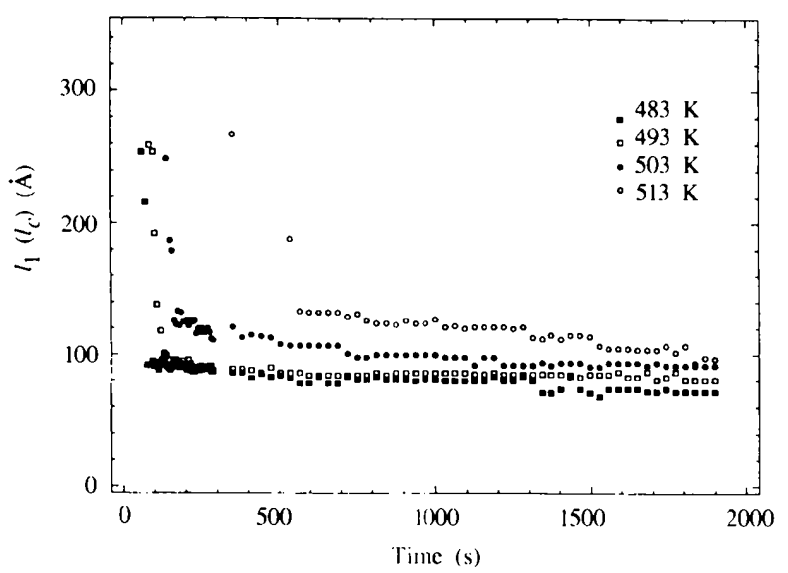

(b)

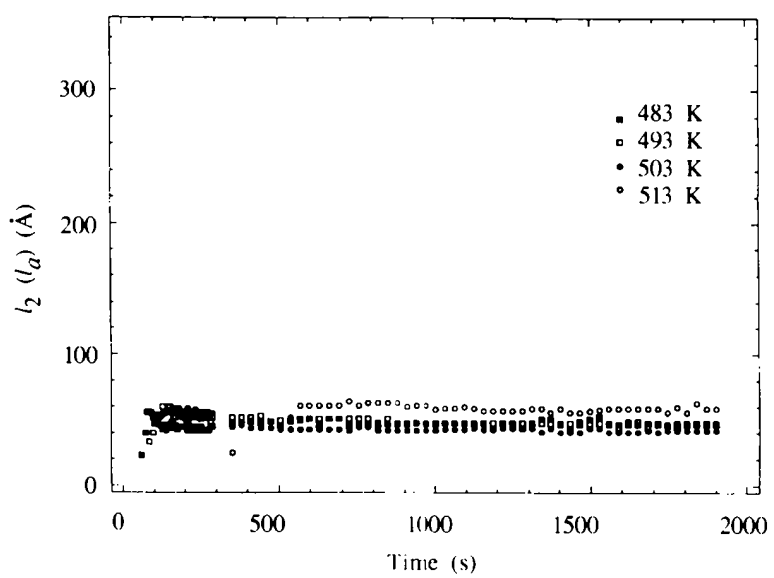

(c)

\section{Figure 8}

Lamellar morphological variables as a function of crystallization temperature: $(a)$ long period $L_{c}^{M},(b)$ crystal lamellar thickness, $l_{c}$, and $(c)$ interlamellar amorphous thickness, $l_{a}$ (the $y$-axis scale was kept at the same range for comparison). behavior is well known in most polymers. Since the effect of $T_{c}$ on $l_{1}$ (Fig. $8 b$ ) is similar to that on $L_{c}^{M}$ but the effect on $l_{2}$ is minimal, it is logical to assign $l_{1}$ (the larger value) as the crystal lamellar thickness. This is because the decrease in the degree of supercooling (higher $T_{c}$ ) should always produce a larger crystal thickness.

\section{Conclusions}

We have demonstrated that the detailed morphological variables in crystalline polymers can be extracted from timeresolved synchrotron SAXS data using a novel approach to calculate the correlation and interface distribution functions. This novel approach is based on two alternative expressions of Porod's law, which provides several advantages over the conventional method. These advantages include the ability to analyze data with a poor signal-to-noise ratio and/or a limited Porod region. An example study of the isothermal crystallization of PET is shown for this purpose. However, we caution that the proposed approach does not add precision nor accuracy to the calculation of correlation and interface distribution functions, which still depends on the noise level and the correct selection of the Porod region. Finally, we add that the correlation and interface distribution functions have to be interpreted with a suitable morphological model. In crystalline polymers we assume that a lamellar two-phase model is representative of the true morphology which may not be true for other systems.

We gratefully acknowledge helpful discussions with Drs A. Biswas, N. Stribeck and the Late Professor H. G. Zachmann for the analysis of correlation and interface distribution functions using the lamellar model. The example SAXS profiles of PET were obtained with the assistance of Drs S. Seifert and P. Harney. This work was supported by DuPont Central Research and Development and a grant from the NSF-GOALI program (DMA9629825).

\section{References}

Baltá-Calleja, F. J. \& Vonk, G. G. (1989). X-ray Scattering of Synthetic Polymers. New York: Elsevier Science.

Chu, B., Harney, P. J., Li, Y., Linliu, K., Yeh, F. \& Hsiao, B. S. (1994). Rev. Sci. Instrum. 65, 597-602.

Debye, P., Anderson, H. R. \& Brauberger, H. (1957). J. Appl. Phys. 28, 679-683.

Debye, P. \& Bueche, A. M. (1949). J. Appl. Phys. 20, 518.

Glatter, O. (1982). Small Angle X-ray Scattering, ch. IV, edited by O. Glatter \& O. Kratky, pp. 167-196. New York: Academic Press.

Hsiao, B. S., Sauer, B. B., Verma, R., Chu, B., Harney, P., Zachmann, H. G. \& Seifert, S. (1995). Macromolecules, 28, 6931-6936.

Koberstein, J. T., Morra, B. \& Stein, R. S. (1980). J. Appl. Cryst. 13, 34-45.

Porod, G. (1951). Kolloid Z. 124, 83-114.

Porod, G. (1952a). Kolloid Z. 125, 51-57.

Porod, G. (1952b). Kolloid Z. 125, 108-122.

Rathje, J. \& Ruland, W. (1976). Collect. Polym. Sci. 254, 358-370. 
Ruland, W. (1977). Collect. Polym. Sci. 255, 417-427.

Russell, T. (1991). Handbook on Synchrotron Radiation, Vol. 3, ch. 11 , edited by G. Brown \& D. E. Moncton, pp. 379-469. New York: Elsevier Science.

Santa Cruz, C., Stribeck, N., Zachmann, H. G. \& Baltá-Calleja, F. J. (1991). Macromolecules, 24, 5980-5990.

Song, H. H., Stein, R. S., Wu, D. Q., Ree, M., Phillips, J. C., LeGrand, A. \& Chu, B. (1988). Macromolecules, 21, 1180.

Stribeck, N. (1993). Collect. Polym. Sci. 271, 1007-1023.

Stribeck, N. \& Ruland, W. (1978). J. Appl. Cryst. 11, 535-539.
Strobl, G. R. \& Schneider, M. (1980). J. Polym. Sci. Polym. Phys. Ed. 18, 1343-1359.

Verma, R. \& Hsiao, B. S. (1996). Trends. Polym. Sci. 4, 312-319.

Vonk, G. G. (1973). J. Appl. Cryst. 6, 81-86.

Vonk, C. G. \& Kortleve, G. (1967). Collect. Polym. Sci. 220, 1924.

Wendorff, J. H. \& Fisher, E. W. (1973a). Kolloid. Z. Z. Polym. 251, 876.

Wendorff, J. H. \& Fisher, E. W. (1973b). Kolloid. Z. Z. Polym. 251, 884. 